\title{
Towards the development of a reliable protocol for mesenchymal stem cell cardiomyogenesis
}

\author{
Faizal Z. Asumda \\ Saint James School of Medicine, Chicago, USA; fasumda@,ovi.com \\ Received 5 October 2012; revised 3 November 2012; accepted 7 December 2012
}

\begin{abstract}
The specific transformative steps that occur from the multipotent through the mature cardiomyocyte state are determined in large part by changes in gene expression. The exact differentiation and developmental programs are tightly regulated in a step-wise systematic fashion based on the influence of specific instigating and signaling factors. Crucial to the observed cell behavior and tissue specific phenotypic differences are alterations in the organization, translocation and expression of nuclear proteins during differentiation. The cardiomyogenic differentiation of Mesenchymal Stem Cells (MSCs) remains a precarious process. Transplanted MSCs must respond to endogenous signaling molecules involved in early embryonic cardiomyogenesis by activating the specific gene regulatory network required for successful cardiomyogenesis and transdifferentiation. To do that, transplanted MSCs would have to be genuinely reprogrammed genetically to become functional cardiac myocytes. A consideration of recent experimental findings suggests that Bone morphogenic protein (BMP-2/4), insulin-like growth factor (IGF-1) and fibroblast growth factor (FGF2) in combination is a potent inducer of MSC cardiomyogenesis. The development of an optimum and reliable in vitro culture milieu for directing their cardiomyogenesis will provide an indispensable model system for molecular studies and genetic manipulation.
\end{abstract}

Keywords: Differentiation; Mesenchymal Stem Cells; Cardiomyogenesis; Cellular Cardiomyoplasty

\section{INTRODUCTION}

Mesenchymal stem cells (MSCs) are a unique population of non-hematopoietic adult stem cells resident in the bone marrow and within various adult tissues. In culture,
MSCs are capable of sustaining extensive proliferative capacity in an uncommitted state. The current modality employed to identify and purify MSCs from co-resident cells is predicated on their expression of a number of surface markers which are in general, not specific to MSCs [1-5]. Overall, MSC populations isolated from different tissue sources express an identical immunophenotype with minute differences in expression levels [3-5]. Bone marrow derived mesenchymal stem cells (BMMSCs) have been extensively used in the clinical setting for cell therapy [4-6]. Irrespective of tissue source, MSCs are purified on the basis of their spindly fibroblast-like morphology, adherence to culture plates, and ability to differentiate into a number of different mesodermal lineages as well positively express a panel of surface markers (CD90, CD105, CD106, CD73, CD44, CD166, CD29, etc.) while remaining negative for markers of hematopoietic and endothelial precursors, lymphocytes and macrophages such as (CD45, CD31, CD34, CD11a, CD14, etc.) [1-5,7-8]. Owing to their multipotent, clonogenic and self-renewing ability, MSCs can be induced in vitro and in vivo to differentiate and transdifferentiate into a variety of mesodermal lineages including osteocytes, chondrocytes, adipocytes, cardiomyogenic cells and neural cells [1-8]. Based on the diversity of surface markers expressed, MSCs are considered a heterogeneous population of cells [4-5]. MSCs are known to express a host of stemness and pluripotent markers, including but not limited to Oct-4, Sox-2, and NANOG, hTERT, Rex-1, KLF4 and cMyc [9-10]. The functional homology of Oct-4 in the presence of other pluripotency genes is identical in MSCs and embryonic stem cells (ESCs) [9]. The expression of ESC markers by MSCs is suggestive of a cell population with extensive proliferation and differentiation capacity [8-11]. MSCs lack the level of pluripotency associated with ESCs, but they maintain robust multipotency; they are therefore less likely to spontaneously differentiate down undesired lineages. This makes them a safer alternative in the clinical setting [6-7]; their engraftment and subsequent transdifferentiation in vivo can be better controlled. MSCs have therefore emerged in recent years as a reliable candidate cell source for stem 
cell therapy. Their use in cellular cardiomyoplasty is extensively documented and studied [4-7].

\section{CARDIOMYOGENIC POTENTIAL OF MESENCHYMAL STEM CELLS}

Functional cardiomyocytes are routinely and efficiently derived from ESCs. In contrast, the cardiomyogenic differentiation of MSCs remains a precarious process. A consideration of experimental findings suggests that MSCs undergoing cardiomyogenesis display varying degrees of the cardiac phenotype [6,11-12]. Most remain skeptical as to if MSCs are capable of attaining the complete repertoire of cardiomyocyte structural and functional characteristics-i.e. the ability to exhibit $\mathrm{Ca}^{2+}$ currents, respond physiologically to cardiaoactive drugs and other stimuli [12-13]. The truth is that MSCs, in vitro, never achieve the level of differentiation typified by adult myocardial cells that provide direct functional benefit in repairing or regenerating myocardium. A key limitation to unlocking their full potential is the field's inability to overcome this hurdle. The primary goal in myocardial regeneration therapy is survival and subsequent transdifferentiation of transplanted cells. Transplanted MSCs are expected to transdifferentiate into functional cardiac myocytes with full contractile and electrophysiological functionality [7]. In theory, transplanted MSCs would first function as authentic in vivo cardiac progenitor cells [14] en route to complete genetic reprogramming to become physiologically functioning cardiac muscle cells. The exact molecular cues that presumably drive this process are yet to be systematically elucidated in vitro; this is painstaking but necessary basic research. Reproducing this phenomenon in an in vitro setting will provide a vital tool for controlled modeling of cardiac disease, drug discovery and testing, genetic manipulation and gene transfer experiments geared at influencing signal transduction, and cardiac function $[12,15]$.

It would appear that the field has seemingly moved beyond this level of systems biological analysis given the extent to which MSCs are being used in the clinical setting [4-6]. A realistic assessment of this burgeoning therapy underscores the fact that the underlying notion that, dead myocardial tissue can be repopulated by simply injecting stem and progenitor cells is a gross oversimplification [14-15]. Positive results in the clinical setting have been modest, owing in large part to the existing knowledge deficit [12,14-15]. Signaling that contributes to MSC cardiomyogenesis remains an important issue given the paucity of information. The development of an optimum and reliable in vitro culture milieu for directing their cardiomyogenesis will provide an indispensable model for molecular studies and genetic manipulation. Such a protocol will enable elucidation of the specific transformative steps that take place from the multipotent state through the cardiovascular progenitor cell state. A number of different approaches have been employed to direct the in vitro cardiomyogenesis of MSCs. Direct co-culture with neonatal ventricular cardiomyocytes (NV $\mathrm{CMs}$ ) and adult cardiomyocytes has been used to induce expression of the cardiac phenotype [8,12-13,16-19]. MSCs co-cultured with NVCMs or adult cardiac myocytes express cardiac connexins, cardiac transcription factors such as Nkx2.5, GATA4, Mef2c, ANP, and cardiac structural proteins (cTnC, cTnT, cTnI, cTm, $\alpha$-sarcomeric actinin, and MHC) $[8,12,16-22]$. Co-culture is inherently self-limiting with regard to being able to uncouple cells for downstream analyses. Furthermore, cell fusion can be erroneously catalogued as transdifferentiation $[8,22]$. Another drawback to co-culture is that, in the absence of direct cell-cell contact, MSCs fail to differentiate [19-20]. Other approaches include electrical stimulation, mechanical stimulation, and pharmacological approaches using oxytocin or 5-azacytidin, fibroblast growth factor (FGF-2) or transforming growth factor (TGF- $\beta$ ) stimulation [13].

5 -azacytidin is a synthetic nucleoside commonly used as an inhibitor of DNA methylation and has been used widely as a treatment strategy to induce MSC cardiomyogenesis. But, it has proven to be inconsistent with reports of positive and negative results [13,23-29]. In instances of success, MSCs treated with 5-azacytidin undergo a fundamental change in morphology. The reported changes include increased size, ball-like or stick-like and myotube-like structures and vigorous beating [24-28]. The exact mechanism of action is still not well understood, but 5-azacytidine has been reported to reactivate MyoD [29]. 5-azacytidine also activates the extracellular regulated kinase (ERK) signaling pathway [28]. The ERK pathway is essential for mesoderm differentiation [30], and mediates osteogenic and adipogenic differentiation of MSCs [31]. But its function in myogenesis remains controversial [32-33]. The key issue with 5-azacytidine is that conflicting results have been reported regarding its inductive effect on MSC cardiomyogenesis. Liu, et al. [23] reported a null effect, Balana, et al. [29] reported a failure to induce expression of MyoD or Nkx2.5 and GATA4 or any cardiac structural proteins, and Takeda, et al. [34] also reported a null effect. 5-azacytidine reversibly inhibits methyltransferase and blocks methylation of DNA [35-36]. The result is the reactivation of previously silenced genes which is thought to be responsible for its inductive effects on various cell types [37]. The mixed results are suggestive of non-specificity in action and in all probability additional factors might be required for consistently reliable results $[12,23]$. The case is stronger for a lack of specificity-5-azacytidine induces phenotypic changes in a variety of cells including fibroblasts to adipocytes and muscle cells [37], erythroid differentia- 
tion to erythroleukemia cells [36], and converts mouse embryonic cell lines into differentiated chondrocytes, adipocytes, and skeletal muscle [35]. Random reprograming of gene expression following 5-azacytidine treatment can not be ruled out [23]. Preadipocytes treated with 5-azacytindine overexpress Nanog, Sox 2 and Oct-4 genes which are associated with self-renewal and pluripotentiality [38]. These data are suggestive of random reprogramming and variability in cell response to 5-azacytidine treatment which makes it unsuitable for clinical use; it is not a reliable and efficient treatment modality.

\section{THE CASE FOR BMP, IGF AND FGF}

The ideal efficient cardiomyogenesis protocol should be developed from a translational perspective-i.e. to facilitate current understanding of in vivo transdifferentiation events, cardiac development and treatment of cardiovascular disease. Early cardiomyogenesis is a complex process, but the activation of the cardiac specific gene program is determined by a number of key cellular signals. Clues can therefore be taken from growth factors (GFs) involved in early embryonic cardiomyogenesis to enhance the derivation of cardiomyocytes from MSCs [39-40]. Bone morphogenic protein (BMP-2/4), insulinlike growth factor (IGF-1) and fibroblast growth factor (FGF-2) are required for cardiac specific lineage commitment in early embryonic development. These three GFs have been extensively screened and tested for their effect on the cardiomyogenesis of ESCs, iPS cells, MSCs, and other adult stem cells [8,11-13,38-57]. BMP functions as part of the TGF- $\beta$ superfamily and its inductive effect on the cardiac gene program is mediated by SMAD phosphorylation and nuclear accumulation of SMADcomplex [41]. The BMP inhibitor, NOG blocks activation of the cardiac gene program in precardiac mesoderm explants and the subsequent formation of contracting cardiomyocytes [47-48]. Of particular importance is BMP$2 / 4$ which mediates activation of the cardiac transcription factors NKX2.5, GATA5, GATA4, MEF2, TBX2, 5, 20 [47-49]. BMP-2/4 is critical for precardioblast formation [48] and enables GATA4 and Nkx2.5 upregulation during cardioblast formation [51-53]. Critical to this endeavor is the knowledge that FGF-2 regulates the differentiation of resident cardiac precursors into cardiomyocytes [54]. Data from Baron, et al. [55] shows that, brief exposure to FGF-2 and contiguous presence of BMP-2 is sufficient to induce cardiac differentiation. Labovsky, et al. [56] further support this conclusion with data showing that neither factor alone is sufficient to induce cardiogenesis in non-precardiac embryonic mesoderm. These specific data show that the action of BMP and FGF in cardiac myocyte formation is cooperative and that timing and length of exposure is of consequence [49,55-56]. IGF-1 is known to mediate the cardioprotective effect of MSCs [58], sup- press apoptosis of cardiomyocytes [59], stimulate contractility, and maintain cardiac myocyte function [60]. IGF-1has also been shown to stimulate increased expression of the gap junction protein connexin-43 in vitro [61], and to enhance the survival and proliferation of cardiac stem cells [62]. Kofidis, et al. [63] demonstrated overexpression of MHC along with enhanced engraftment and transdifferentiation of ESCs in host myocardium following supplementation with IGF-1.

The main distinguishing feature between MSCs and ESCs is the level of pluripotency. In terms of pluripotency genes, MSCs are known to express, the array of stemness markers seen in ESCs [8-11]; the functional homology of these genes in MSCs is identical to that seen in ESCs [9]. Identical transcription factors trigger activation of the cardiac gene program in both ESCs and adult stem cells [16,64-65]. From a theoretical standpoint, it is expected that the role of BMP-2/4, FGF-2 and IGF-1 in the cardiac differentiation of ESCs will be identical in MSCs (Figure 1). Presumably, undifferentiated multipotent MSCs will first undergo a precardioblast or early cardiac progenitor stage marked by diminished expression of stemness genes and recapitulation of the embryonic differentiation program $[12,41,66]$. It is presumed here that, MSCs at this stage will express primary early cardiac transcription factors such as Nkx2.5, GATA4, Mef2c, Tbx5/20 and Hand1/2 [16,41,64-65]. It is also presumed that MSCs at this stage of transformation may yet express either low levels of stemness markers such as OCT4 and SOX-2 or null expression might be the case. Following this stage, MSCs are expected to undergo a cardioblast or early cardiomyocyte stage which is presumably marked by definitively high levels of nuclear GATA4, and Nkx2.5 along with varying levels of structural and sarcomeric proteins such as MHC, cTn, cTm and $\alpha$-actinin $[12,15,41,65]$. The mature cardiomyocyte stage will be marked by full expression of the cardiac gene program along with the full repertoire of structural and myofilament lattice proteins and most importantly will presumably be characterized by mature myofibrillar organization, calcium handling and electrophysiology $[12$, 41,66].

The case and ensuing evidence for a combination treatment of MSCs with BMP-2, FGF-2 and IGF-1 has been made [7-8,11-13,45-46]. Asumda, et al. [8,11] induced BM-MSC cardiomyogenesis following treatment with DMEM supplemented with 5\% FBS, $50 \mathrm{ng} \cdot \mathrm{ml}^{-1} \mathrm{FGF}-2$, $5 \mathrm{ng} \cdot \mathrm{ml}^{-1} \mathrm{IGF}-1$, and $20 \mathrm{ng} \cdot \mathrm{ml}^{-1} \mathrm{BMP}-2$. It is worth noting here that the specific BM-MSC population used by Asumda, et al. $[8,11]$ expressed high levels of the ESC markers OCT4, SOX-2, and NANOG and demonstrated extensive multi-differentiation capacity. BM-MSCs induced with this treatment strategy fail to display the spontaneous syncytial contraction or striations typical of 


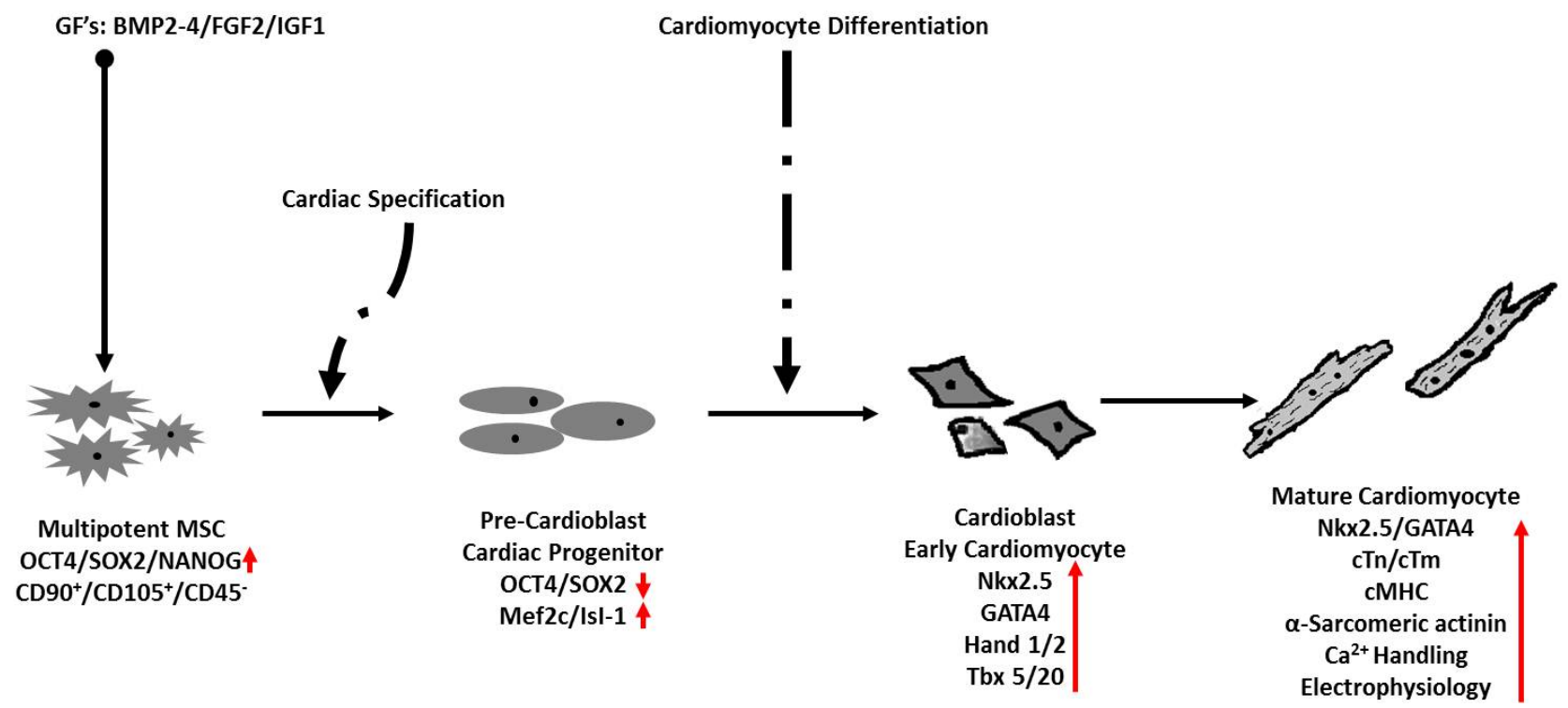

Figure 1. Illustration of expected stages of cardiomyogenesis in MSCs following GF treatment. Multipotent MSCs expressing Oct4, SOX2 and NANOG commit to the cardiac lineage following induction by forming cardiac progenitors. Cells at this stage are proliferative, express significantly high levels of early cardiac transcription factors such as Isl-1 and MEF and possibly low levels of Oct4/SOX2. Continued induction will activate expression of cardiomyocyte-enriched transcription factors such as Nkx2.5, GATA4, Hand $1 / 2$ and Tbx 5/20. Cells at this stage are early cardiomyocytes that will go on to express sarcomeric and structural proteins, and demonstrate functional activity synonymous with mature contracting cardiomyocytes.

isolated neonatal and adult ventricular cardiomyocytes. But they undergo dramatic morphological change from the spindly-fibroblast appearance typical of MSCs. Progressive enlargement and elongation with formation of ball and stick-like morphology; binucleation and the formation of long vacuolated cytoplasmic processes with distinct surface characteristics are observed. Also observed are connections between adjoining cells, and formation of myotube-like structures. It is worth noting that these observations shown in Figure 2 were also reported elsewhere [24-28] following 5-azacytidine treatment. A full array of cardiac transcription factors and structural proteins including GATA4, Nkx2.5, GATA6, MHC, connexin $43, \alpha$-sarcomeric actinin, cTnT, cTnI cTnC, cTm are also detectable $[9,11]$. These observations are remarkably reproducible and are consistent with data from others [45-46] using an identical treatment strategy. Of importance is the fact that the GF's are required in combination [45-46] and individually, they fail to induce the nuclear translocation of key transcription factors such as Nkx2.5, GATA4 and Mef2C [45].

It is important to note that the specific transformative steps that occur, from the multipotent through the mature cardiomyocyte state is determined almost exclusively by changes in gene expression. The exact differentiation and developmental programs are tightly regulated in a stepwise systematic fashion based on the influence of specific instigating and signaling factors; recapitulation of the events of embryonic cardiac differentiation by MSCs is far from arbitrary. Crucial to the observed cell behavior and tissue specific phenotypic differences are alterations in the organization, translocation and expression of nuclear proteins during differentiation. Presumably, the same applies to MSCs transplanted to the injured myocardium. Transplanted MSCs are expected to respond to endogenous signaling molecules involved in early embryonic cardiomyogenesis within host myocardium by activating the specific gene regulatory network required for successful cardiomyogenesis and transdifferentiation. To do that, transplanted MSCs would have to be genuinely reprogrammed genetically to become functional cardiomyocytes as opposed to taking on the behavior of native cells with which they are fused [14]. Such an occurrence is contingent first on the in vitro demonstration that, MSCs do in fact differentiate into physiologically functional, stable, specialized cardiac muscle cells [14]. The nuclear expression of $\mathrm{cTn}$ and $\mathrm{cTm}$ by BM-MSCs undergoing cardiomyogenesis is discussed and examined in greater detail elsewhere [7,11]. It is worth noting here that, GATA4 and Nkx2.5 act synergistically to activate cardiac transcription [67] and furthermore, cardiac differentiation of MSCs is driven by the nuclear translocation of GATA4 and Nkx2.5 [65]. Shown in Figure 3 is the nuclear expression of Nkx2.5 and GATA4 early in the cardiomyogenesis of treated BM-MSCs. In connection with this, BMP-2/4 and FGF-2 are known to target, activate and maintain GATA4 and Nkx2.5 expression [50, $51,54]$. 

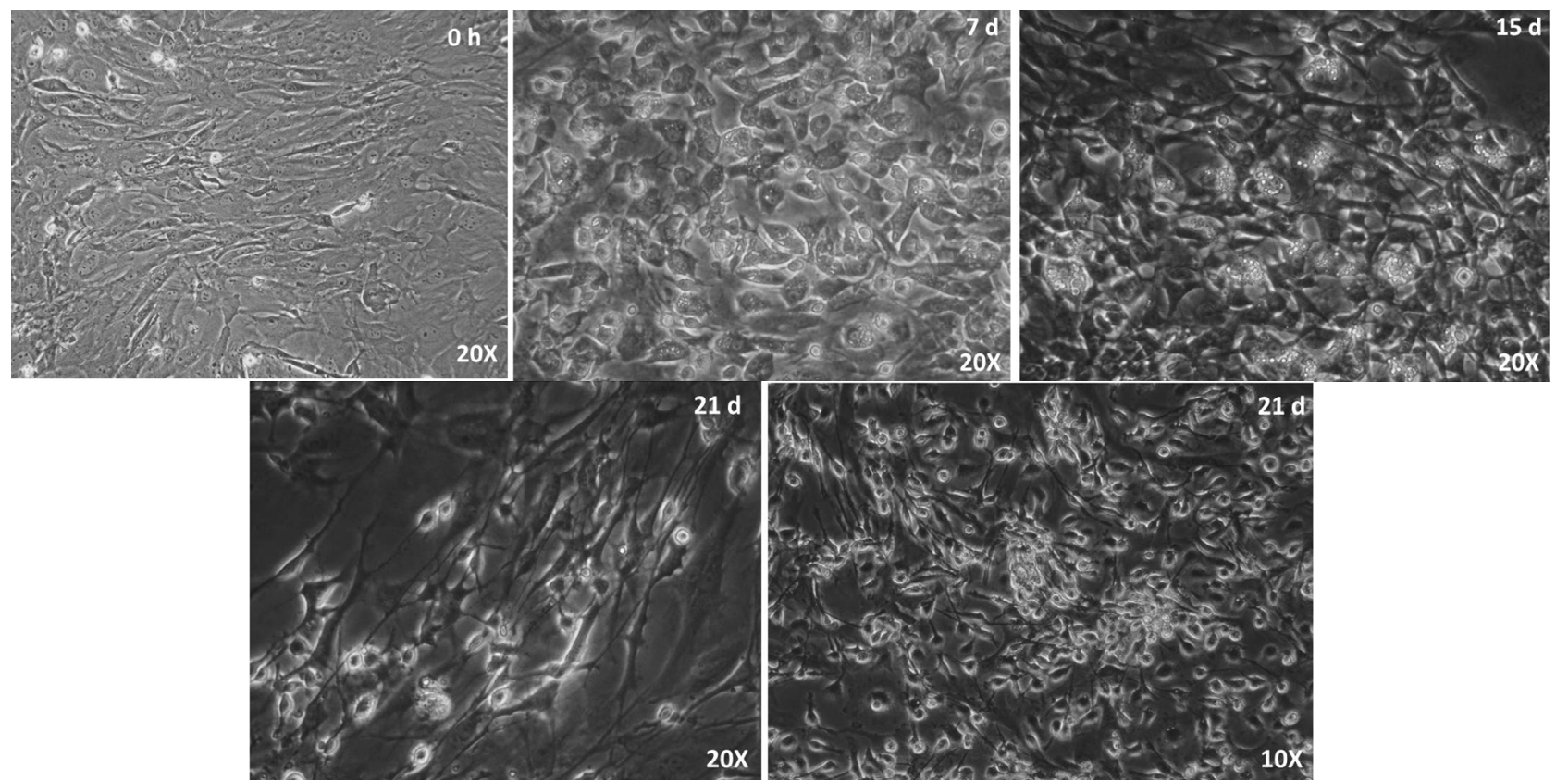

Figure 2. Phase Contrast Micrographs of cardiomyogenic cells. MSCs show typical spindly-fibroblast like morphological before GF treatment. A dramatic change in morphology is observed 7 days ( 7 d) after treatment. Progressive enlargement and elongation, ball and stick- like morphology and long vacuolated cytoplasmic processes with distinct surface characteristics are observed. Cardiomyogenic cells appear stretched and spherical, connect with adjoining cells and form distinct combinations of myoblast and myotube-like cells at 15 days $(15 \mathrm{~d})$ and 21 days $(21 \mathrm{~d})$ post induction.
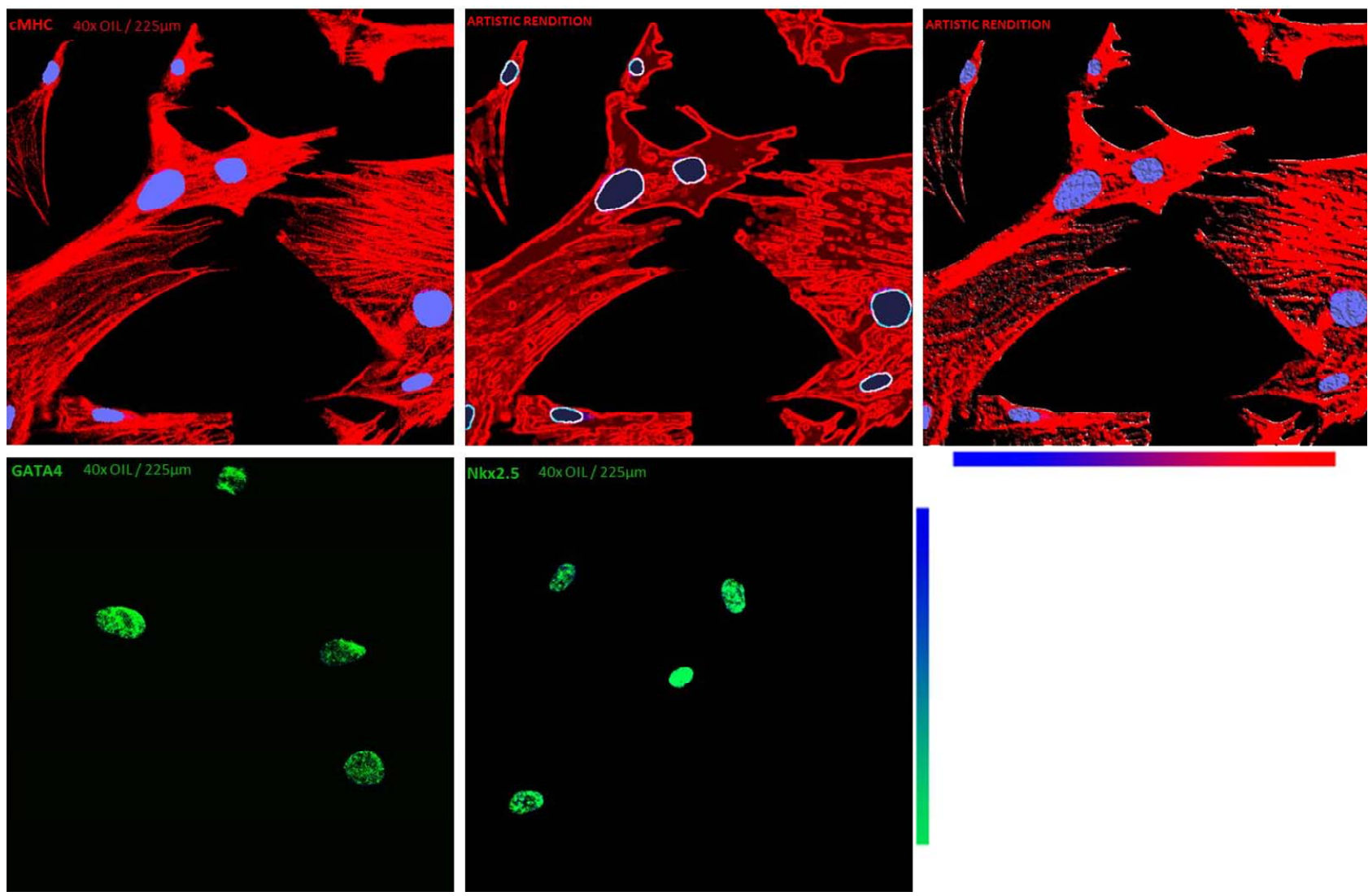

Figure 3. Immunocytochemical demonstration of cMHC (top row), GATA4 (bottom left) and Nkx2.5 (bottom right) expression in MSCs undergoing cardiomyogenesis. 


\section{DISCUSSION}

In comparison to the data presented here and reported by Asumda, et al. [7-8,11], few studies have been as successful at inducing the simultaneous expression of this many cardiac specific transcription factors and structural proteins in MSCs. While, MSCs have thus far failed to achieve the level of differentiation synonymous with native ventricular cardiomyocytes, their ability to recapitulate the embryonic cardiac developmental pathway and gene program under the influence of these three GFs (BMP-2, FGF-2 and IGF-1) provides a reproducible in vitro model system for further mechanistic studies. It is unclear what the missing link is at this stage. But the goal still remains - that is, to generate morphological and functional cardiomyocytes from MSCs with syncytial and electro-mechanical contractile ability synonymous with their endogenous counterparts in a consistent and reproducible manner. Given the heterogeneous nature of MSCs a number of issues may arise. Extensive culture over multiple population doublings might result in the selection of MSC populations with significantly higher intrinsic expression of cardiomyogenic genes $[57,68]$. Bayes-Genis, et al. [68] previously reported that, extensive in vitro culture promotes the selection of MSC subpopulations with high intrinsic expression of cardiomyogenic genes in the absence of inductive stimuli. The specific cells used by Asumda, et al. $[8,11]$ were negative for cardiac markers in the absence of inductive media. Furthermore, characterization of the exact number of cells positive for the observed cardiac markers was not assessed by Asumda, et al. [8,11]. It is highly likely that within a specific MSC population, a significant number of cells might fail to activate the cardiac genome. But given time and continued induction, all cells are expected to undergo differentiation. Asumda, et al. $[8,11]$ also induced adipogenesis in their BM-MSCs; over the course of three weeks, all cells committed and differentiated into fully functional adipocytes $[8,11]$. The existence of authentic MSCs in the bone marrow already committed to the cardiac lineage raises a host of questions. It suggests that, the cardiac microenvironment is favorable for their commitment to the cardiac lineage. It also indicates that in the absence of cardiac specific characterization of isolated MSCs, existent subpopulations within what is inherently a heterogeneous population might be more susceptible to in vitro cardiac differentiation stimuli. The heterogeneity within a specific population, differences between isolated populations and interspecies differences are issues inherent in the definition of what MSCs are [5]. It lends credence to the view that MSCs are a phenomenon of in vitro culture [5]. It further highlights the need to substantiate, reproduce and correlate hypothetical in vivo transdifferentiation events either by transplanted
MSCs or endogenous MSCs that traffic to the heart following injury [69] within a controlled in vitro system.

\section{CONCLUSION}

Despite the recent flurry of studies employing a variety of protocols to induce MSC cardiomyogenesis, the process remains unreliable at best. Development of a welldefined and efficient cardiomyogenic protocol should be done with clinical applications in mind; it should utilize safe and proven recombinant cytokines and growth factors. Taken together, the available data support the theoretical exposition that combination treatment with BMP, IGF and FGF is a potentially effective and reliable treatment modality for derivation of cardiomyocytes from MSCs.

\section{REFERENCES}

[1] Pittenger, M.F., Mackay, A.M., Beck, S.C., Jaiswal, R.K., Douglas, R., Mosca, J.D., Moorman, M.A., Simonetti, D.W., Craig, S. and Marshak, D.R. (1999) Multilineage potential of adult human mesenchymal stem cells. Science, 284, 143-147. doi:10.1126/science.284.5411.143

[2] Deans, R.J. and Moseley, A.B. (2000) Mesenchymal stem cells: Biology and potential clinical uses. Experimental Hematology, 28, 875-884. doi:10.1016/S0301-472X(00)00482-3

[3] Kolf, C.M., Cho, E. and Tuan, R.S. (2007) Mesenchymal stromal cells. Biology of adult mesenchymal stem cells: Regulation of niche, self-renewal and differentiation. Arthritis Research \& Therapy, 9, 204-215. doi:10.1186/ar2116

[4] Williams, A.R. and Hare, J.M. (2011) Mesenchymal stem cells: Biology, pathophysiology, translational findings, and therapeutic implications for cardiac disease. Circulation Research, 109, 923-940. doi:10.1161/CIRCRESAHA.111.243147

[5] Keating, A. (2012) Mesenchymal stromal cells: New directions. Cell Stem Cell, 10, 709-716. doi:10.1016/j.stem.2012.05.015

[6] Heldman, A.W., Zambrano, J.P. and Hare, J.M. (2011) Cell therapy for heart disease: Where are we in 2011? Journal of the American College of Cardiology, 57, 466468. doi:10.1016/j.jacc.2010.09.028

[7] Asumda, F. (2012) Bone marrow mesenchymal stem aging: Implications for cellular cardiomyoplasty: A theoretical exposition. Lambert Academic Publishing, Saarbrücken.

[8] Asumda, F.Z. and Chase, P.B. (2012) Age-related changes in rat bone-marrow mesenchymal stem cell plasticity. BMC Cell Biology, 12, 44-55. doi:10.1186/1471-2121-12-44

[9] Greco, S.J., Liu, K. and Rameshwar, P. (2007) Functional similarities among genes regulated by Oct 4 in human mesenchymal and embryonic stem cells. Stem Cells, 25, 31433154. doi:10.1634/stemcells.2007-0351 
[10] Gonzalez, R., Maki, C.B., Pacchiarotti, J., Csontos, S., Pham, J.K., Slepko, N., Patel, A. and Silva, F. (2007) Pluripotent marker expression and differentiation of human second trimester mesenchymal stem cells. Biochemical and Biophysical Research Communications, 362, 491-497. doi:10.1016/j.bbrc.2007.08.033

[11] Asumda, F.Z. and Chase, P.B. (2012) Nuclear cardiac troponin and tropomyosin are expressed early in cardiac differentiation of rat mesenchymal stem cells. Differentiation, 83, 106-115. doi:10.1016/j.diff.2011.10.002

[12] Young, D.A., DeQuach, J.A. and Christman, K.L. (2011) Human cardiomyogenesis and the need for systems boilogy analysis. Wiley Interdisciplinary Reviews Systems Biology and Medicine, 3, 666-680. doi:10.1002/wsbm.141

[13] Heng, B.C., Haider, H.K., Sim, E.K., Cao, T. and Ng, S.C. (2004) Strategies for directing the differentiation of stem cells into the cardiomyogenic lineage in vitro. Cardiovascular Research, 62, 34-42. doi:10.1016/j.cardiores.2003.12.022

[14] Chien, K.R. (2004) Stem cells: Lost in translation. Nature, 428, 607-608. doi:10.1038/nature02500

[15] Chien, K.R. (2008) Regenerative medicine and human models of human disease. Nature, 453, 302-305. doi:10.1038/nature07037

[16] Armiñán, A., Gandía, C., García-Verdugo, J.M., Lledó, E., Mullor, J.L., Montero, J.A. and Sepúlveda, P. (2010) Cardiac transcription factors driven lineage-specification of adult stem cells. Journal of Cardiovascular Translational Research, 3, 61-65. doi:10.1007/s12265-009-9144-3

[17] Nishiyama, N., Miyoshi, S., Hida, N., Uyama, T., Okamoto, K., Ikegami, Y., Miyado, K., Segawa, K., Terai, M., Sakamoto, M., et al. (2007) The significant cardiomyogenic potential of human umbilical cord blood-derived mesenchymal stem cells in vitro. Stem Cells, 25, 20172024. doi:10.1634/stemcells.2006-0662

[18] Spees, J.L., Olson, S.D., Ylostalo, J., Lynch, P.J., Smith, J., Perry, A., Peister, A., Wang, M.Y. and Prockop, D.J. (2003) Differentiation, cell fusion, and nuclear fusion during ex vivo repair of epithelium by human adult stem cells from bone marrow stroma. Proceedings of the $\mathrm{Na}$ tional Academy of Sciences of the United States of America, 100, 2397-2402. doi:10.1073/pnas.0437997100

[19] Labovsky, V., Hofer, E.L., Feldman, L., Fernández, V.V., García, R.H., Bayes-Genis, A., Hernando, I.A., Levin, M.J. and Chasseing, N.A. (2010) Cardiomyogenic differentiation of human bone marrow mesenchymal cells: Role of cardiac extract from neonatal rat cardiomyocytes. Differentiation, 79, 93-101. doi:10.1016/j.diff.2009.10.001

[20] Valiunas, V., Doronin, S., Valiuniene, L., Potapova, I., Zuckerman, J., Walcott, B., Robinson, R.B., Rosen MR, Brink, P.R. and Cohen, I.S. (2004) Human mesenchymal stem cells make cardiac connexins and form functional gap junctions. The Journal of Physiology, 555, 617-626. doi:10.1113/jphysiol.2003.058719

[21] Ramkisoensing, A., Pijnappels, D., Schalij, M., de Vries, A. and Atsma, D. (2012) Connexin43 expression is essential for functional cardiomyogenic differentiation of human fetal mesenchymal stem cells. Journal of the
American College of Cardiology, 59, E855. doi:10.1016/S0735-1097(12)60856-0

[22] Garbade, J., Schubert, R., Lenz, D., Walther, T., Gummert, J., Dhein, S. and Mohr, F.W. (2005) Fusion of bone marrow-derived stem cells with cardiomyocytes in a heterologous in vitro model. European Journal of CardioThoracic Surgery, 28, 685-691. doi:10.1016/j.ejcts.2005.06.047

[23] Liu, Y., Song, J., Liu, W., Wan, Y., Chen, X. and Hu, C. (2003) Growth and differentiation of rat bone marrow stromal cells: Does 5-azacytidine trigger their cardiomyogenic differentiation? Cardiovascular Research, 58, 460468. doi:10.1016/S0008-6363(03)00265-7

[24] Rangappa, S., Fen, C., Lee, E.H., Bongso, A. and Sim, E.K. (2000) Transformation of adult mesenchymal stem cells isolated from the fatty tissue into cardiomyocytes. Annals of Thoracic Surgery, 75, 775-779. doi:10.1016/S0003-4975(02)04568-X

[25] Antonitsis, P., Papagiannaki, E.I., Kaidoglou, A. and Papakonstantinou, C. (2007) In vitro cardiomyogenic differentiation of adult human bone marrow mesenchymal stem cells: The role of 5-azacytidine. Interactive Cardiovascular and Thoracic Surgery, 6, 593-597. doi:10.1510/icvts.2007.157875

[26] Zhang, Y., Chu, Y., Shen, W. and Dou, Z. (2009) Effect of 5 -azacytidine induction duration on differentiation of human first-trimester fetal mesenchymal stem cells towards cardiomyocyte-like cells. Interactive Cardiovascular and Thoracic Surgery, 9, 943-946. doi:10.1510/icvts.2009.211490

[27] Makino, S., Fukuda, K., Miyoshi, S., Konishi, F., Kodama, H., Pan, J., Sano, M., Takahashi, T, Hori, S. and Abe, H., et al. (1999) Cardiomyocytes can be generated from marrow stromal cells in vitro. The Journal of Clinical Investigation, 103, 697-705. doi:10.1172/JCI5298

[28] Qian, Q., Qian, H., Zhang, X., Zhu, W., Yan, Y., Ye, S., Peng, X., Li, W., Xu, Z., Sun, L. and Xu, W. (2012) 5-Azacytidine induces cardiac differentiation of human umbilical cord-derived mesenchymal stem cells by activating extracellular regulated kinase. Stem Cells Development, 21, 67-75. doi:10.1089/scd.2010.0519

[29] Balana, B., Nicoletti, C., Zahanich, I., Graf, E. M., Christ, T., Boxberger, S. and Ravens, U. (2006) 5-Azacytidine induces changes in electrophysiological properties of human mesenchymal stem cells. Cell Research, 16, 949-960. doi:10.1038/sj.cr.7310116

[30] Yao, Y., Li, W., Wu, J., Germann, U.A., Su, M.S., Kuida, K. and Boucher, D.M. (2003) Extracellular signal-regulated kinase 2 is necessary for mesoderm differentiation. Proceedings of the National Academy of Sciences of the United States of America, 28, 12759-12764. doi:10.1073/pnas.2134254100

[31] Jaiswal, R.K., Jaiswal, N., Bruder, S.P., Mbalaviele, G., Marshak, D.R. and Pittenger, M.F. (2000) Adult human mesenchymal stem cell differentiation to the osteogenic or adipogenic lineage is regulated by mitogen-activated protein kinase. Journal of Biological Chemistry, 275, 9645-9652. doi:10.1074/jbc.275.13.9645

[32] Kim, H.S., Cho, J.W., Hidaka, K. and Morisaki, T. (2007) 
Activation of MEK-ERK by heregulin-betal promotes the development of cardiomyocytes derived from ES cells. Biochemical and Biophysical Research Communications, 28, 732-738. doi:10.1016/j.bbrc.2007.07.045

[33] Tortorella, L.L., Milasincic, D.J. and Pilch, P.F. (2001) Critical proliferation-independent window for basic fibroblast growth factor repression of myogenesis via the $\mathrm{p} 42$ / p44 MAPK signaling pathway. Journal of Biological Chemistry, 276, 13709-13717. doi:10.1002/jgm.583

[34] Takeda, Y., Mori, T., Imabayashi, H., Kiyono, T., Gojo, S., Miyoshi, S., Hida, N., Ita, M., Segawa, K. and Ogawa, S. (2004) Can the life span of human marrow stromal cells be prolonged by bmi-1, E6, E7, and/or telomerase without affecting cardiomyogenic differentiation? Journal of Gene Medicine, 6, 833-845.

[35] Taylor, S.M. and Jones, P.A. (1982) Mechanism of action of eukaryotic DNA methyltransferase. Use of 5-azacytosine-containing DNA. Journal of Molecular Biology, 162, 679-692. doi:10.1016/0022-2836(82)90395-3

[36] Creusot, F., Acs, G. and Christman, J.K. (1982) Inhibition of DNA methyltransferase and induction of Friend erythroleukemia cell differentiation by 5-azacytidine and 5aza-29-deoxycytidine. Journal of Biological Chemistry, 257, 2041-2048.

[37] Taylor, S.M. and Jones, P.A. (1979) Multiple new phenoltypes induced in 10T1/2 and 3T3 cells treated with 5-azacytidine. Cell, 17, 771-779. doi:10.1016/0092-8674(79)90317-9

[38] Cifarelli, R.A., Conconi, M.T., Marmo, R., Liddo, R., Dininno, C., Ferraro, S., Cellini, F. and Parnigotto, P. (2012) 5-Azacytidine makes human preadipocytes able to differentiate into mesoderm-derived cell lineages. Stem Cells Development, 21, 76-85. doi:10.1089/scd.2010.0464

[39] Fishman, M.C. and Chien, K.R. (1997) Fashioning the vertebrate heart: Earliest embryonic decisions. Development, 124, 2099-2117.

[40] Roura, S., Farré, J., Hove-Madsen, L., Prat-Vidal, C., Soler-Botija, C., Gálvez-Montón, C., Vilalta, M. and Bayes-Genis, A. (2010) Exposure to cardiomyogenic stimuli fails to transdifferentiate human umbilical cord bloodderived mesenchymal stem cells. Basic Research in Cardiology, 105, 419-430. doi:10.1007/s00395-009-0081-8

[41] van Wijk, B., Moorman, A.F. and van den Hoff, M.J. (2007) Role of bone morphogenetic proteins in cardiac differentiation. Cardiovascular Research, 74, 244-255. doi:10.1016/j.cardiores.2006.11.022

[42] Kawai, T., Takahashi, T., Esaki, M., Ushikoshi, H., Nagano, S., Fujiwara, H. and Kosai, K. (2004) Efficient cardiomyogenic differentiation of embryonic stem cells by fibroblast growth factor 2 and bone morphogenetic protein. Circulation, 68, 691-702. doi:10.1253/circj.68.691

[43] Bartunek, J., Croissant, J.D., Wijns, W., Gofflot, S., de Lavareille, A., Vanderheyden, M., Kaluzhny, Y., Mazouz, N., Willemsen, P. and Penicka, M. (2007) Pretreatment of adult bone marrow mesenchymal stem cells with cardiomyogenic growth factors and repair of the chronically infarcted myocardium. American Journal of Physiology. Heart and Circulatory Physiology, 292, H1095-H1104.

\section{doi:10.1152/ajpheart.01009.2005}

[44] Laflamme, M.A., Chen, K.Y., Naumova, A.V., Muskheli, V., Fugate, J.A., Dupras, S.K., Reinecke, H., Xu, C., Hassanipou, M. and Police, S. (2007) Cardiomyocytes derived from human embryonic stem cells in pro-survival factors enhance function of infarcted rat hearts. Nature Biotechnology, 25, 1015-1024. doi:10.1038/nbt1327

[45] Behfar, A., Yamada, S., Crespo-Diaz, R., Nesbitt, J.J., Rowe, L.A., Perez-Terzic, C., Gaussin, V., Homsy, C., Bartunek, J. and Terzic, A. (2010) Guided cardiopoiesis enhances therapeutic benefit of bone marrow human mesenchymal stem cells in chronic myocardial infarction. Journal of American College of Cardiology, 56, 722-734. doi:10.1016/j.jacc.2010.03.066

[46] Hahn, J.Y., Cho, H.J., Kang, H.J., Kim, T.S., Kim, M.H., Chung, J.H., Bae, J.W., Oh, B.H., Park, Y.B. and Kim, H.S. (2008) Pre-treatment of mesenchymal stem cells with a combination of growth factors enhances gap junction formation, cytoprotective effect on cardiomyocytes, and therapeutic efficacy for myocardial infarction. Journal of American College of Cardiology, 51, 933-943. doi:10.1016/j.jacc.2007.11.040

[47] Burridge, P. W., Thompson, S., Millrod, M., Weinberg, S., Yuan, X., Peters, A., Mahairaki, V., Koliatsos, V.E., Tung, L. and Zambidis, E.T., (2011) A universal system for highly efficient cardiac differentiation of human induced pluripotent stem cells that eliminates interline variability. PloS One, 6, e18293. doi:10.1371/journal.pone.0018293

[48] Redig, J.K. and Adler, E. (2011) Doing the dirty work: Progress in the search for a reliable protocol for cardiomyogenesis. Stem Cell Research \& Therapy, 2, 35. doi:10.1186/scrt76

[49] Schlange, T., Andree, B., Arnold, H.H. and Brand, T. (2000) BMP2 is required for early heart development during a distinct time period. Mechanisms of Development, 91, 259-270. doi:10.1016/S0925-4773(99)00311-1

[50] Schultheiss, T.M., Burch, J.B. and Lassar, A.B. (1997) A role for bone morphogenetic proteins in the induction of cardiac myogenesis. Genes \& Development, 11, 451-462. doi:10.1101/gad.11.4.451

[51] Monzen, K., Nagai, R. and Komuro, I. (2002) A role for bone morphogenetic protein signaling in cardiomyocyte differentiation. Trends in Cardiovascular Medicine, 12, 263-269. doi:10.1016/S1050-1738(02)00172-X

[52] Sugi, Y., Yamamura, H., Okagawa, H. and Markwald, R.R. (2004) Bone morphogenetic protein-2 can mediate myocardial regulation of atrioventricular cushion mesenchymal cell formation in mice. Developmental Biology, 269, 505-518. doi:10.1016/j.ydbio.2004.01.045

[53] Qi, X., Li, T.G., Hao, J., Hu, J., Wang, J. and Simmons, H. (2004) BMP4 supports self-renewal of embryonic stem cells by inhibiting mitogen-activated protein kinase pathways. Proceedings of the National Academy of Sciences of the United States of America, 101, 6027-6032. doi:10.1073/pnas.0401367101

[54] Rosenblatt-velin, N., Lepore, M.G., Cartoni, C., Beermann, F. and Pedrazzini, T. (2005) FGF-2 controls the differentiation of resident cardiac precursors into functional cardiomyocytes. The Journal of Clinical Investiga- 
tion, 115, 1724-1733. doi:10.1172/JCI23418

[55] Barron, M., Gao, M. and Lough, J. (2000) Requirement for BMP and FGF signaling during cardiogenic induction in non-precardiac mesoderm specific, transient, and cooperative. Developmental Dynamics, 218, 383-393. doi:10.1002/(SICI)1097-0177(200006)218:2<383::AIDDVDY11>3.0.CO;2-P

[56] Lough, J., Barron, M., Brogley, M., Sugi, Y., Bolender, D.L. and Zhu, X. (1996) Combined BMP-2 and FGF-4, but neither factor alone, induces cardiogenesis in non-precardiac embryonic mesoderm. Developmental Biology, 178, 198-202. doi:10.1006/dbio.1996.0211

[57] Siegel, G., Krause, P., Wöhrle, S., Nowak, P., Ayturan, M., Kluba, T., Brehm, B.R., Neumeister, B., Köhler, D. and Rosenberger, P. (2012) Bone marrow-derived human mesenchymal stem cells express cardiomyogenic proteins but do not exhibit functional cardiomyogenic differentiation potential. Stem Cells Development. Epub Ahead of Print. doi:10.1089/scd.2011.0626

[58] Sadat, S., Gehmert, S., Song, Y.H., Yen, Y., Bai, X., Gaiser, S., Klein, H. and Alt, E. (2007) The cardioprotective effect of mesenchymal stem cells is mediated by IGF-I and VEGF. Biochemical and Biophysical Research Communications, 363, 674-679. doi:10.1016/j.bbrc.2007.09.058

[59] Lee, W.L., Chen, J.W., Ting, C.T., Ishiwata, T., Lin, S.J., Korc, M. and Wang, P.H. (1999) Insulin-like growth factor I improves cardiovascular function and suppresses apoptosis of cardiomyocytes in dilated cardiomyopathy. Endocrinology, 140, 4831-4840. doi:10.1210/en.140.10.4831

[60] Solem, M.L. and Thomas, A.P. (1998) Modulation of cardiac Ca2+ channels by IGF1. Biochemical and Biophysical Research Communications, 252, 151-155. doi:10.1006/bbrc.1998.9626

[61] Aberg, N.D., Blomstrand, F., Aberg, M.A., Björklund, U., Carlsson, B., Carlsson-Skwirut, C., Bang, P., Rönnbäck, L. and Eriksson, P.S. (2003) Insulin-like growth factor-I increases astrocyte intercellular gap junctional communication and connexin43 expression in vitro. Journal of Neuroscience Research, 74, 12-22. doi:10.1002/jnr.10734

[62] Urbanek, K., Rota, M., Cascapera, S., Bearzi, C., Nascimbene, A., De Angelis, A., Hosoda, T., Chimenti, S., Baker, M. and Limana, F. (2005) Cardiac stem cells pos-

\section{List of Abbreviations}

ANP, atrial natriuretic peptide;

BM-MSC, bone marrow-derived mesenchymal stem cells;

BMP-2, bone morphogenetic protein 2;

cTnI, cardiac troponin I;

cTnT, cardiac troponin $\mathrm{T}$;

cTnC, cardiac troponin C;

cTrpm, cardiac tropomyosin;

DMEM, Dulbecco's modified Eagle's medium; sess growth factor-receptor systems that after activation regenerate the infarcted myocardium, improving ventricular function and long-term survival. Circulation Research, 97, 663-673.

doi:10.1161/01.RES.0000183733.53101.11

[63] Kofidis, T., de Bruin, J.L., Yamane, T., Balsam, L.B., Lebl, D.R. and Swijnenburg, R.J. (2004) Insulin-like growth factor promotes engraftment, differentiation, and functional improvement after transfer of embryonic stem cells for myocardial restoration. Stem Cells, 22, 1239 1245. doi:10.1634/stemcells.2004-0127

[64] Perez-Terzic, C., Faustino, R. S., Boorsma, B. J., Arrell, D. K., Niederlander, N. J., Behfar, A. and Terzic, A. (2007) Stem cells transform into a cardiac phenotype with remodeling of the nuclear transport machinery. Nature Clinical Practice Cardiovascular Medicine, 1, S68-S76. doi:10.1038/ncpcardio0763

[65] Arminan, A., Gandia, C., Bartual, M., Garcia-Verdugo, J.M., Lledo, E., Mirabet, V., Llop, M., Barea, J., Montero, J.A. and Sepúlveda, P. (2009) Cardiac differentiation is driven by NKX2.5 and GATA4 nuclear translocation in tissue-specific mesenchymal stem cells. Stem Cells and Development, 18, 907-918. doi:10.1089/scd.2008.0292

[66] Naito, A.T., Tominaga, A., Oyamada, M., Oyamada, Y., Shiraishi, I., Monzen, K., Komuro, I. and Takamatsu, T. (2003) Early stage-specific inhibitions of cardiomyocyte differentiation and expression of Csx/Nkx-2.5 and GATA4 by phosphatidylinositol 3-kinase inhibitor LY294002. Experimental Cell Research, 291, 56-69. doi:10.1016/S0014-4827(03)00378-1

[67] Durocher, D., Charron, F., Warren, R., Schwartz, R.J. and Nemer, M. (1997) The cardiac transcription factors Nkx25 and GATA-4 are mutual cofactors. The EMBO Journal, 16, 5687-5696. doi:10.1093/emboj/16.18.5687

[68] Bayes-Genis, A., Roura, S., Soler-Botija, C., Farré, J., Hove-Madsen, L., Llach, A and Cinca, J. (2005) Identification of cardiomyogenic lineage markers in untreated human bone marrow-derived mesenchymal stem cells. Transplantation Proceedings, 37, 4077-4079. doi:10.1016/j.transproceed.2005.09.103

[69] Dulce, R., Balkan, W., Hare, J.M. and Schulman, I.H. (2011) Wnt signalling: A mediator of the heart-bone marrow axis after myocardial injury? European Heart Journal, 2, 2-4.

FBS, fetal bovine serum;

FGF-2, fibroblast growth factor 2;

IGF-1, insulin-like growth factor 1;

iPS, induced pluripotent stem cells;

MEF, myocyte specific enhancer factor;

cMHC, cardiac myosin heavy chain;

MSCs, mesenchymal stem cells;

NVCM, neonatal ventricular cardiomyocytes 